\title{
PHRF1 promotes genome integrity by modulating non-homologous end-joining
}

\author{
C-F Chang ${ }^{1,3}$, P-C Chu ${ }^{1,3}$, P-Y Wu ${ }^{1}$, M-Y Y $u^{2}$, J-Y Lee ${ }^{2}$, M-D Tsai ${ }^{1,2}$ and M-S Chang ${ }^{*, 1,2}$
}

Methylated histone readers are critical for chromatin dynamics, transcription, and DNA repair. Human PHRF1 contains a plant homeodomain (PHD) that recognizes methylated histones and a RING domain, which ubiquitinates substrates. A recent study reveals that PHRF1 is a tumor suppressor that promotes TGF- $\beta$ cytostatic signaling through TGIF ubiquitination. Also, PHRF1 is a putative phosphorylation substrate of ataxia telangiectasia-mutated/ataxia telangiectasia and Rad3-related kinases; however, the role of PHRF1 in DNA damage response is unclear. Here we report a novel function of PHRF1 in modulating non-homologous endjoining (NHEJ). PHRF1 quickly localizes to DNA damage lesions upon genotoxic insults. Ablation of PHRF1 decreases the efficiency of plasmid-based end-joining, whereas PHRF1 overexpression leads to an elevated NHEJ in H1299 reporter cells. Immunoprecipitation and peptide pull-down assays verify that PHRF1 constitutively binds to di- and trimethylated histone H3 lysine 36 (H3K36) (H3K36me2 and H3K36me3) via its PHD domain. Substitution of $S^{915} D^{917} E$ to ADAE in PHRF1 decreases its affinity for NBS1. Both PHD domain and SDTE motif are required for its NHEJ-promoting activity. Furthermore, PHRF1 mediates PARP1 polyubiquitination for proteasomal degradation. These results suggest that PHRF1 may combine with H3K36 methylation and NBS1 to promote NHEJ and stabilize genomic integrity upon DNA damage insults.

Cell Death and Disease (2015) 6, e1716; doi:10.1038/cddis.2015.81; published online 9 April 2015

Unrepaired double-strand breaks (DSBs) can lead to oncogenic transformations or cell death. In higher eukaryotes, DSBs are mainly repaired by homologous recombination (HR; error-free repair) and non-homologous end-joining (NHEJ; error-prone repair). ${ }^{1,2}$ It has been suggested that DSB response mainly relies on the $\gamma$-H2AX-MDC1-RNF8-RNF168 axis, but diverges to BRCA1 for HR and 53BP1 for NHEJ. ${ }^{1,2}$ The definitive step for HR or NHEJ is dependent on Rap1interacting factor 1 (Rif1). In the absence of Rif1, DNA ends undergo BRCA1-dependent resection to facilitate HR. In the G1 phase, Rif1 in association with the phosphorylated 53BP1 protects DNA end resection mediated by BRCA1-CtIP and consequently promotes NHEJ. ${ }^{3-6}$ Classical NHEJ mainly occurs in the G1 phase. Ku70/Ku80 heterodimer binds to DNA ends and recruits DNA-PKcs, Artemis, and Pol $\mu / \lambda$ to the repair site, resulting in end processing followed by ligase IV, XRCC4, and XLF-mediated ligation. ${ }^{7}$ In the absence of Ku proteins, Mre11-RAD50-NBS1 (MRN) in conjunction with CtIP facilitate alternative NHEJ (alt-NHEJ) using short microhomologies ${ }^{8}$. Furthermore, MRN recognizes DSBs and promotes HR during the S/G2 phase. ${ }^{8}$

Histone methylation has key roles in chromatin remodeling and regulation of gene transcription, DNA replication, genome stability, and apoptosis. ${ }^{9,10}$ Histone lysine residues can be monomethylated (me1), dimethylated (me2), or trimethylated (me3) on particular histone methylation sites, such as histone H3 lysine 4 (H3K4), H3K9, H3K27, H3K36, H3K79, and H4K20. ${ }^{9,10}$ Methylated histones are recognized by readers containing specific domains, such as plant homeodomain (PHD), WD40 repeat, chromodomain, and Tudor domain. ${ }^{11-16}$ Readers that recognize methylated histones could recruit other chromatin remodeling enzymes to render cis- or transmodifications on histones, thus regulating chromatin dynamics and controlling cellular functions.

Several studies have reported the connection between histone methylation and NHEJ. Notably, 53BP1 recruitment to DNA lesions is through the binding of its tudor domain with increased dimethylated H4K20 (H4K20me2) at local DNA damage sites during NHEJ. ${ }^{17,18}$ The methylation of H4K20me2 by histone methyltransferase MMSET in mammals is critical for the recruitment of 53BP1 to DSBs. ${ }^{19}$ However, this scenario has been argued by other groups because they could not detect any defects in 53BP1 recruitment in MMSET-deficient cells. Instead, H3K79me2 is required for 53BP1 foci formation during the G1/G2 phases while the level of H4K20me2 is low. ${ }^{20}$ Recently, H3K36me2 has been demonstrated to be involved in NHEJ pathway. Locally increased H3K36me2 improves the association of

\footnotetext{
${ }^{1}$ Institute of Biological Chemistry, Academia Sinica, Taipei 11529, Taiwan and ${ }^{2}$ Institute of Biochemical Sciences, College of Life Science, National Taiwan University, Taipei 11529, Taiwan

*Corresponding author: M-S Chang, Institute of Biochemical Sciences, National Taiwan University, No. 1, Section 4, Roosevelt Road, Taipei 10617, Taiwan. Tel: +886 23366 9837; Fax: +886 22363 5038; E-mail: mschang@ntu.edu.tw

${ }^{3}$ These authors contributed equally to this work.

Abbreviations: Alt-NHEJ, alternative non-homologous end-joining; ATM, ataxia telangiectasia-mutated; ATR, ataxia telangiectasia and Rad3-related; BrdU, 5-bromo-2deoxyuridine; ChIP, chromatin immunoprecipitation; CPT, camptothecin; CSR, class-switch recombination; DSB, double-strand break; H3K36, histone H3 lysine 36; H3k36me2, dimethylated H3K36; H3K36me3, trimethylated H3K36; H4K2Ome2, dimethylated H4K20; HR, homologous recombination; MMR, DNA mismatch repair; MRN, Mre11-RAD50-NBS1; NHEJ, non-homologous end-joining; PHD, plant homeodomain; Rif1, Rap1-interacting factor 1; SLE, systemic lupus erythematosus; SNP, singlenucleotide polymorphisms; SSc, systemic sclerosis; Ub, ubiquitin

Received 17.6.14; revised 3.2.15; accepted 2.3.15; Edited by D Heery
} 
Ku70 and NBS1 and promotes DSB repair by NHEJ, possibly mediated by an unidentified histone reader protein. ${ }^{21}$

Ataxia telangiectasia-mutated (ATM) and ataxia telangiectasia and Rad3-related (ATR) kinases are important signaling kinases in DNA damage response. ${ }^{1,2}$ Using anti-pSQ/pTQ antibody to perform a large-scale identification of ATM and ATR-phosphorylated targets, PHRF1 is found to be phosphorylated on S925 and S1389 in response to ionizing irradiation. $^{22}$ The primary structure shows that PHRF1 contains one RING domain (amino acid (a.a.) 106-150) for E3 ligase activity, one PHD domain (a.a. 185-233) for association with methylated histones, one putative CK2 phosphorylation motif (SDTE, a.a. 915-918), and seven $\mathrm{SQ} / \mathrm{TQ}$ motifs mainly located on its $C$ terminus. Very recently, PHRF1 is demonstrated as a tumor suppressor responsible for the ubiquitination of TGIF to release $\mathrm{CPML}$ and promote the TGF- $\beta$ cytostatic program. ${ }^{23}$ In an effort to illustrate the function of PHRF1 in DNA damage response, we analyzed cellular responses to genotoxic treatments in PHRF1knockdown and -overexpressing cells. Our results suggest that PHRF1 associates with methylated H3K36 and interacts with NBS1 to promote NHEJ in response to genotoxic stress.

\section{Results}

PHRF1 binds to DNA lesions upon genotoxic stress. As PHRF1 is an ATM/ATR-phosphorylated substrate, we set out to examine whether PHRF1 associated with chromatin in response to genotoxic stress. Subcellular fractionation showed that PHRF1 was mainly present in soluble nuclear extracts without camptothecin (CPT, a topoisomerase I inhibitor) treatment. In contrast, the amount of PHRF1 was elevated in the chromatin fraction after CPT treatment (Figure 1a). To visualize the chromatin association of PHRF1, HeLa cells were exposed to CPT and then pre-extracted with $0.5 \%$ Triton X-100. Triton-extracted cells also showed an increased chromatin-associated PHRF1 after CPT exposure (Figure 1b).

To further confirm that PHRF1 was recruited to DNA damage sites, we monitored the in vivo recruitment of GFPPHRF1 in response to laser-induced DNA lesions. Interestingly, live-cell imaging revealed a fast accumulation of PHRF1 at the irradiated sites followed by a rapid decrease reaching to the basal fluorescence level, with a peaking at $90 \mathrm{~s}$ and lasting to 5 min after laser irradiation. By contrast, GFP-MDC1, a key mediator in DNA damage response that recognizes $\gamma$-H2AX signal, exhibits a much slower recruitment than that of PHRF1 under the same laser irradiation, appearing after $90 \mathrm{~s}$ and remaining accumulated to the end of experiment (Figure 1c). Quantitatively, the protein fluorescence at laser-irradiation site shows distinct recruitment kinetics of PHRF1 and MDC1, in which PHRF1 appeared at DNA damage sites more quickly but transiently compared with MDC1 (Figure 1d). These results likely suggest that the recruitment of PHRF1 is a very early event of DNA damage response. In agreement, PHRF1 did not localize to MDC1-associated foci at $2 \mathrm{~h}$ after laser irradiation in GFP-MDC1 cells (Supplementary Figure S1) nor colocalize with any of $\gamma$-H2AX, RNF8 or BRCA1 following $3 \mathrm{~h}$ CPT treatment in HeLa cells (Supplementary Figure S1). This transient recruitment behavior possibly resulted in the exclusion of PHRF1 from the $\gamma$-H2AX- and MDC1-associated nuclear foci upon longer postincubation time.

PHRF1 affects cell viability in response to genotoxic insults. To investigate whether PHRF1 protects the chromosome integrity, we analyzed chromosome lesions using the comet assay. The majority of PHRF1-depleted U2OS cells exposed to etoposide exhibited damaged and fragmented DNA with distinct tails. By contrast, most of the control cells did not have similar DNA tails (Figure 2a). Conversely, PHRF1overexpressing U2OS cells migrated with normal patterns, but $\sim 80 \%$ of control U2OS cells had fragmented DNA tails (Figure 2b). We examined whether PHRF1 rendered cells resistance to genotoxic insults by clonogenic assay. PHRF1depleted U2OS cells barely survived after CPT or etoposide treatments. By contrast, PHRF1-overexpressing U2OS cells had much higher viability than control cells after genotoxic treatments (Figure 2c). Similar results for comet and clonogenic assays were revealed in PHRF1-depleted and -overexpressing HeLa cells and also in breast cancer MDA-MB-231 cells (Supplementary Figure S2). We measured the effects of PHRF1 on cell cycle progression by flow cytometry. The addition of CPT in PHRF1knockdown and -overexpressing cells did not result in accumulated phases in cell cycle progression. Instead, an increased subG1 population in PHRF1-depleted U2OS cells and a reduced sub-G1 population in PHRF1-overexpressing U2OS cells exposed to CPT suggested that PHRF1 is required for cell viability upon genotoxic insults. Again, similar results were found in PHRF1-depleted HeLa and MDA-MB-231 cells (Supplementary Figure S3). We further analyzed the expression of cleaved caspase-3 to confirm whether PHRF1 affected cell viability via apoptosis. The degraded and activated product of caspase-3 was increased in PHRF1-depleted cells, whereas this apoptotic marker was decreased in PHRF1-overexpressing cells after UV irradiation or CPT exposure (Figure 2d). Collectively, these data suggested that PHRF1 modulates apoptosis upon genotoxic stress.

PHRF1 does not affect DNA damage signaling response. To investigate whether PHRF1 affects DNA damage response, we examined the phosphorylation levels of RPA2 (a singlestrand binding protein) and $\gamma-\mathrm{H} 2 \mathrm{AX}$ (a marker for DSB) in the presence or absence of PHRF1. There was no significant change in phosphorylated RPA2, $\gamma-\mathrm{H} 2 \mathrm{AX}$, Chk1 at S345, and Chk2 at T68 in PHRF1-depleted cells. Total amounts of RNF8 and RNF168 were also unchanged (Supplementary Figure S4). Additionally, the foci formation of RNF8, 53BP1, and BRCA1 was not affected in PHRF1-depleted and -overexpressing cells (Supplementary Figure S4), suggesting that PHRF1 may not impair DNA damage response.

We speculated that HR repair might be modulated by PHRF1. Nonetheless, the depletion and overexpression of PHRF1 did not alter the formation of rad51 foci (Supplementary Figure S5) and the proportion of GFP-positive cells in HR reporter DR-GFP cells was slightly changed in PHRF1depleted and -overexpressing cells (Supplementary Figure S5), indicating that PHRF1 might not affect the efficiency of HR.

PHRF1 associates with histone H3K36me2 and H3K36me3. Immunoprecipitation and immunoblotting analyses showed that 
a

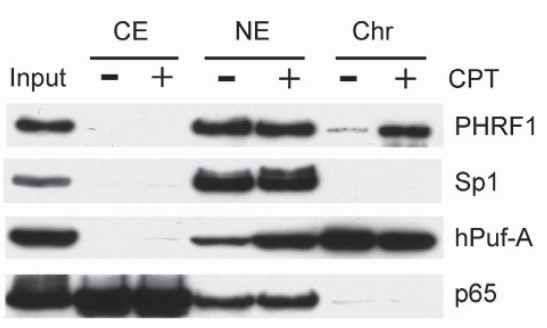

b

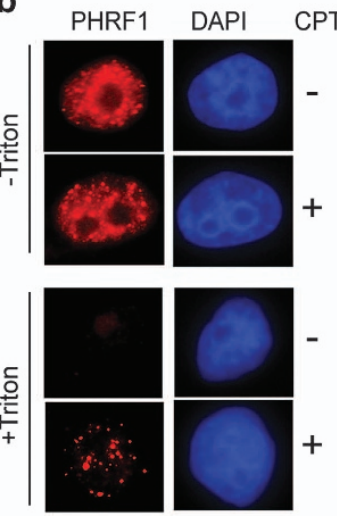

C

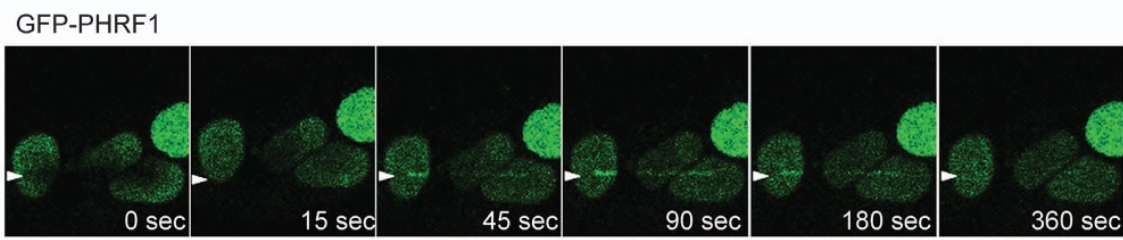

GFP-MDC1

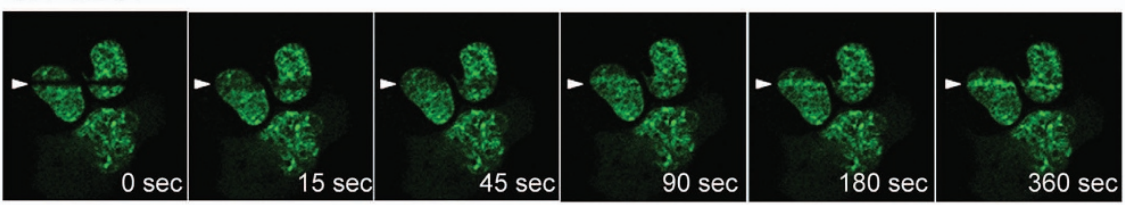

d

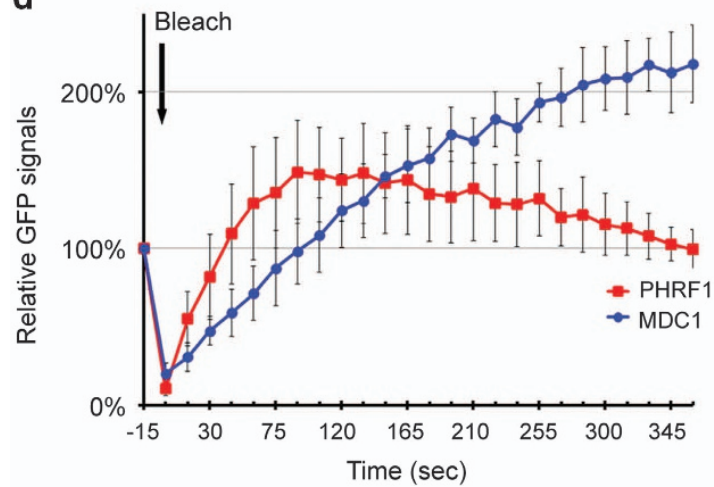

Figure 1 PHRF1 associates with chromatin after genotoxic stress. (a) Subcellular fractionation of HeLa cell extracts was prepared for immunoblot analysis. P65 is a subunit of nuclear factor- $\kappa \mathrm{B}(\mathrm{NF}-\kappa \mathrm{B})$ as a CE marker. Sp1 is an NE marker. hPuf-A, a nucleolar protein moving from chromatin to nuclear extracts upon genotoxic stress. (b) HeLa cells were pre-extracted by cytoskeleton extraction buffer containing $0.5 \%$ Triton X-100 and fixed in paraformaldehyde for immunofluorescence (bar $=10 \mu \mathrm{m})$. (c) Dynamic recruitment of GFP-PHRF1 to DNA damage sites generated by laser microirradiation in U2OS cells. GFP-MDC1 was a control. (d) Kinetics of the recruitment of GFP-PHRF1 and GFP-MDC1 to DNA damage sites were analyzed from 10 microirradiated cells in each experiment. The highest GFP intensity was calculated as $100 \%$ and data were presented as mean \pm S.D. $\mathrm{CE}$, cytosolic extracts; Chr, chromatin-enriched fraction; GFP, green fluorescent protein; NE, nuclear extracts; WCE, whole-cell extracts

PHRF1 is associated with $\mathrm{H} 3 \mathrm{~K} 36 \mathrm{me} 2$ and $\mathrm{H} 3 \mathrm{~K} 36 \mathrm{me} 3$. A timecourse study on immunoprecipitates of PHRF1 showed that the association of PHRF1 with $\mathrm{H} 3 \mathrm{~K} 36 \mathrm{me} / 3$ was increased at 0.5 and $1 \mathrm{~h}$ but declined at $1 \mathrm{~h}$ after CPT exposure. (Figure $3 \mathrm{a}$ ). It has been suggested that an undefined reader protein might recognize H3K36me2 and H3K36me3 near DSBs to promote the binding of MRN complex and Ku70 for NHEJ repair. ${ }^{24}$ It is most likely that the elevated association of PHRF1 with H3K36me2/3 is due to locally increased H3K36me2/3 upon DNA damage, which has been suggested in $\gamma$-irradiation. ${ }^{24}$ Endogenous PHRF1 was specifically pulled down by biotin-labeled $\mathrm{H} 3 \mathrm{~K} 36 \mathrm{me} 3$ peptide in vitro, but not by other peptides (Figure 3b). Interestingly, HA-PHRF1 purified from HEK293T cells could associate with biotin-labeled H3K36me2 and H3K36me3 (data not shown), suggesting that PHRF1 may interact with H3K36me2 under certain circumstances. When HeLa cells were simultaneously labeled with anti-PHRF1, anti-H3K36me2, and anti-H3K36me3 antibodies, the image showed large amount of overlapping areas in the nucleus (Figure 3c). By contrast, PHRF1 was not immunoprecipitated by other methylated histones including H3K4, H3K9, H3K27, H4K20 and acetylated histones (Supplementary Figure 
a

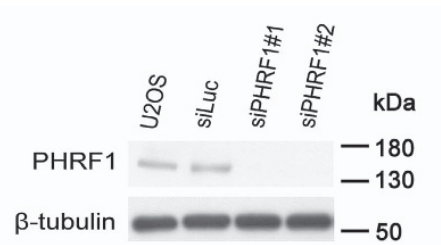

b
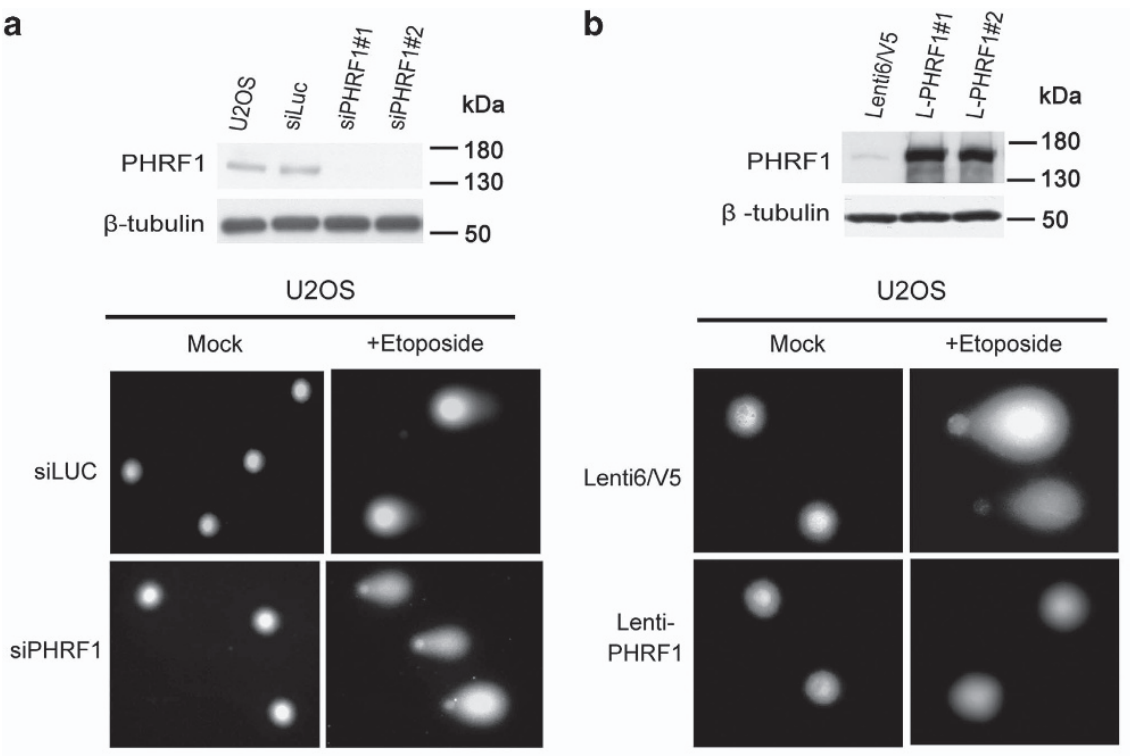

c
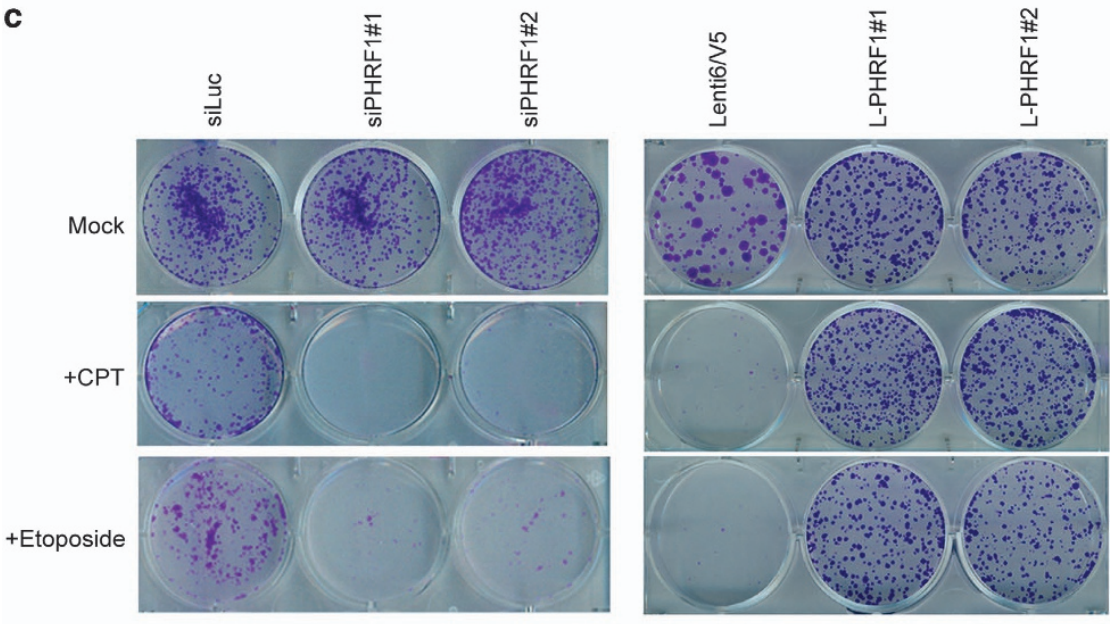

U2OS
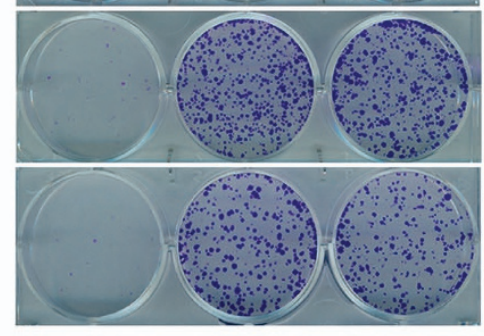

U2OS

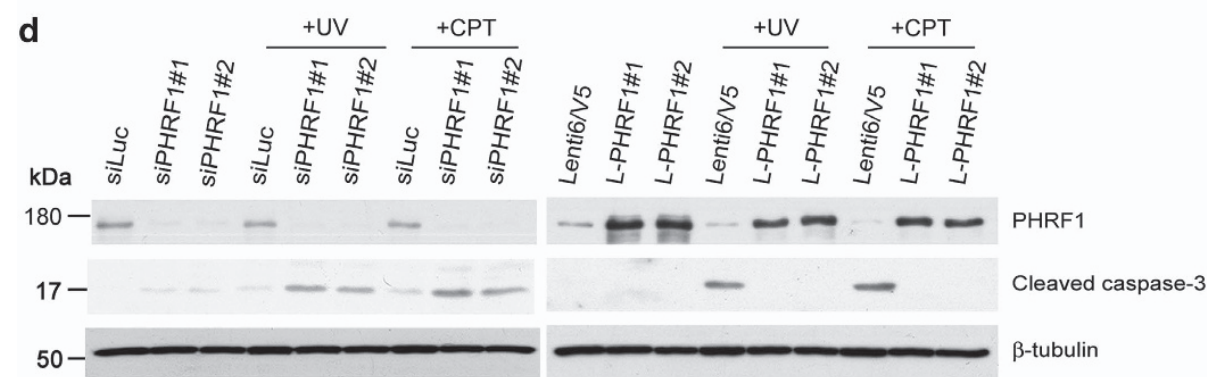

Figure 2 PHRF1 protects cells from genotoxic insults. (a) PHRF1-depleted U2OS cells were exposed to etoposide for 30 min and then collected for the comet assay. (b) Control and PHRF1-overexpressing U2OS cells were exposed to etoposide for $1 \mathrm{~h}$ and then collected for the comet assay. (c) PHRF1-depleted U2OS cells were exposed to CPT $(2 \mu \mathrm{M})$ for $24 \mathrm{~h}$ or etoposide $(10 \mu \mathrm{M})$ for $1 \mathrm{~h}$ and PHRF1-overexpressing cells were treated with CPT $(5 \mu \mathrm{M})$ for $24 \mathrm{~h}$ or etoposide $(25 \mu \mathrm{M})$ for $1 \mathrm{~h}$ for clonogenic assays. Colonies were allowed to form for 10 days and stained with crystal violet. (d) PHRF1-depleted and -overexpressing cells were treated with CPT (10 $\mu \mathrm{M})$ and UV light $\left(20 \mathrm{~J} / \mathrm{m}^{2}\right)$. Total cell extracts were collected after $2 \mathrm{~h}$ and immunoblotted with anticleaved caspase- 3 antibody to examine apoptosis

S6). Additionally, PHRF1 did not colocalize with these methylated histones as revealed by immunofluorescence analysis (Supplementary Figure S6).

Subsequently, to determine which region of PHRF1 is responsible for its association with $\mathrm{H} 3 \mathrm{~K} 36 \mathrm{me}$, HA-tagged
PHRF1 and its deletion mutants lacking RING, PHD, and SQ/TQ motifs were transfected into HEK293T cells. Immunoprecipitation by anti-HA agarose followed by immunoblotting analysis showed that PHD domain, but not RING or SQ/TQ motifs, is required for association with H3K36me2 and 
a

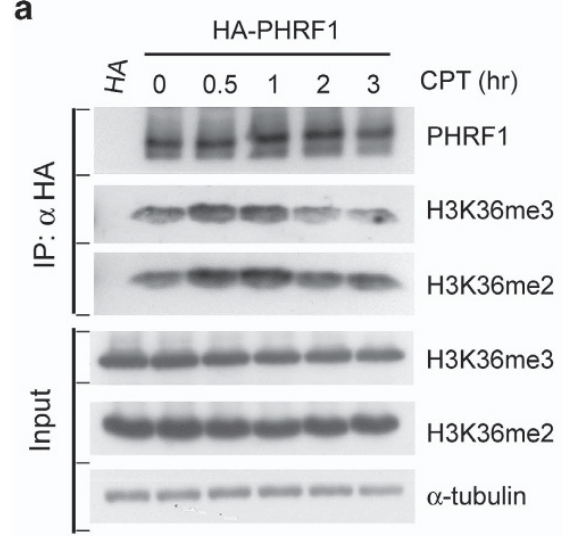

C
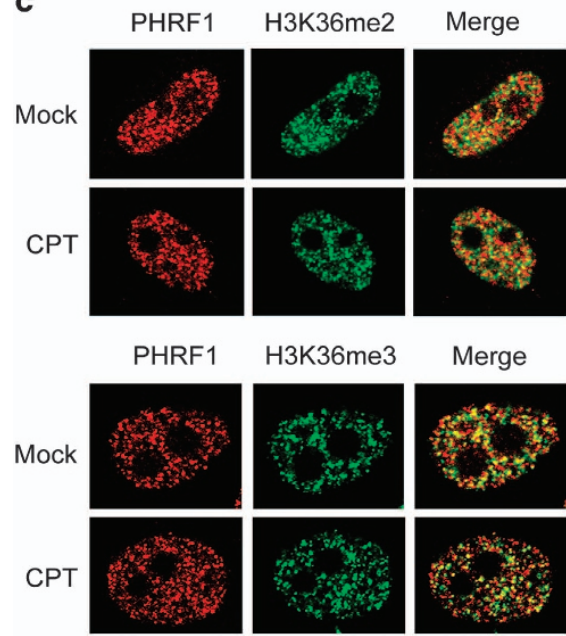

H3K36me3

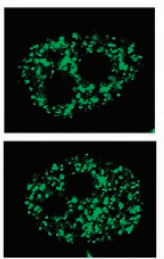

b

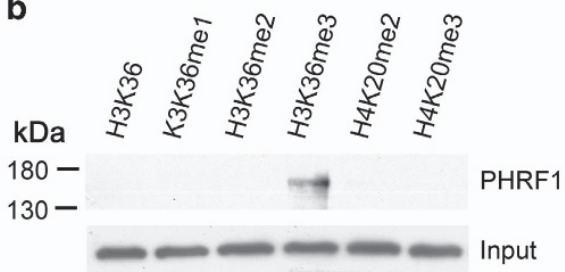

d
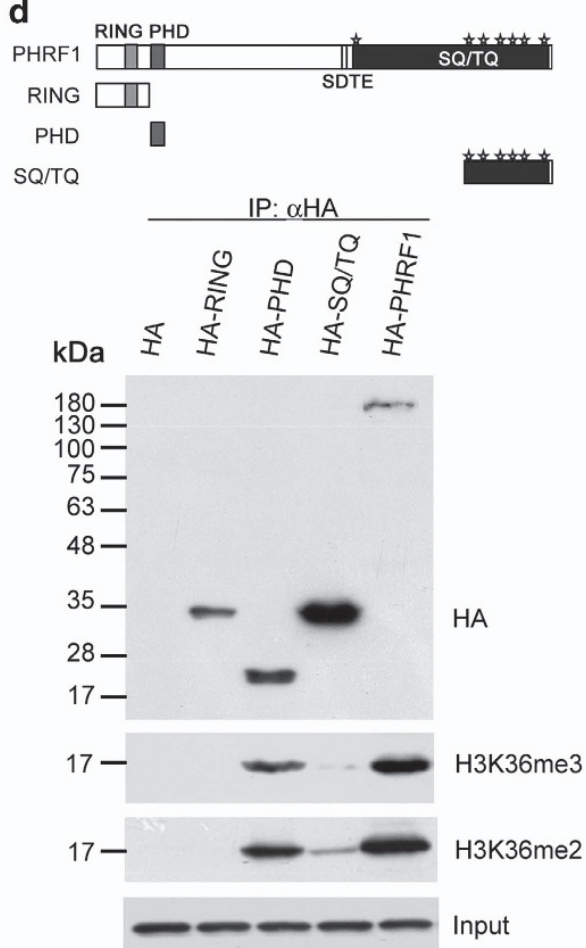

Figure 3 PHRF1 associates with H3K36me2 and H3K36me3. (a) Empty hemagglutinin (HA) vector and HA-PHRF1 were transfected into HEK293T cells for $48 \mathrm{~h}$ and cell extracts were immunoprecipitated (IP) with anti-HA agarose. Immunoblotting analysis was conducted using indicated anti-methyl histone antibodies. (b) Synthetic biotin-labeled H3K36-methylated peptides were incubated with HeLa cell extracts. Immunoblotting analysis was carried out using anti-PHRF1 antibody. (c) HeLa cells were simultaneously labeled with a mixture of anti-PHRF1 monoclonal antibody (mAb) (red) and anti-H3K36me2 or anti-H3K36me3 polyclonal antibodies (green). (d) HA-RING, HA-PHD, HA-SQ/TQ motif, and HA-PHRF1 were transfected into HEK293T cells and immunoprecipitated with anti-HA agarose. Immunoblotting was carried out using indicated antibodies. Note that there are seven SQ/TQ motifs in PHRF1

H3K36me3 (Figure 3d). Collectively, PHRF1 is a methylated histone reader to recognize $\mathrm{H} 3 \mathrm{~K} 36 \mathrm{me} 2$ and $\mathrm{H} 3 \mathrm{~K} 36 \mathrm{me} 3$ via its PHD domain.

PHRF1 affects NHEJ in vivo. To address whether PHRF1 is involved in linking methylated histone to NHEJ, a Hindlllinearized pGL3 plasmid containing the luciferase gene was transfected into PHRF1-depleted HEK293T cells and PHRF1-overexpressing U2OS cells, respectively. The results showed that end-joining activity was significantly reduced in PHRF1-knockdown cells, but increased in PHRF1-overexpressing cells compared with control cells (Figures $4 \mathrm{a}$ and b). Additionally, using a GFP-based NHEJ reporter H1299 human lung cancer cell harboring IRES-TK-EGFP DNA integrated in the genome, ${ }^{25}$ the proportion of EGFPpositive cells was decreased in PHRF1-knockdown cells, but increased in PHRF1-overexpressing cells after I-Scel transfection (Figures $4 \mathrm{c}$ and $\mathrm{d}$ ). These data further support that PHRF1 modulates NHEJ in vivo.
SDTE motif is required for the association of PHRF1 with NBS1. SDTE motif is a conserved CK2 phosphorylation site and may interact with the FHA domain of NBS1. ${ }^{26-28}$ As PHRF1 contains one SDTE motif, we set out to investigate whether PHRF1 modulates NHEJ activity by interacting with NBS1. Immunoprecipitation followed by immunoblotting analysis showed that PHRF1 and NBS1 were in the same immunocomplex. PHRF1 predominantly associated with NBS1 and little amount of Mre11 was in the precipitates of PHRF1 (Figure 5a). Consistent with this, coimmunostaining studies of HeLa cells treated with $10 \mu \mathrm{M}$ CPT for $2 \mathrm{~h}$ revealed a partial colocalization of PHRF1 with NBS1 (Figure 5b). To elucidate the mechanistic interaction between PHRF1 and NBS1, we substituted SDTE to S915A/T917A in PHRF1

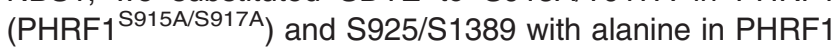

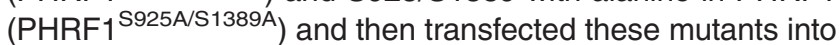
HEK293T cells. All substitution mutants of PHRF1 were listed in Supplementary Figure S7. As anticipated, a mutant PHRF1 protein in which S915 and T917 were substituted with alanine 
showed significantly reduced association with NBS1, whereas S925A and S925A/S1389A mutants did not alter the formation of this complex (Figure $5 \mathrm{c}$ ). Furthermore, using synthetic peptides labeled with biotin, we found that only $\mathrm{pS}^{915} \mathrm{DpT}^{917} \mathrm{E}$ double-phosphorylated peptide, but not single phosphorylated or unphosphorylated peptides, could pull down endogenous NBS1 (Figure 5d).

Furthermore, DSBs were generated by I-Scel digestion in NHEJ reporter $\mathrm{H} 1299$ cells and chromatin immunoprecipitation (ChIP) by anti-NBS antibody was conducted. PCR analysis to detect a $0.3 \mathrm{~kb}$ region downstream of the second I-Scel site showed an enrichment of NBS1 and PHRF1 in this region after a
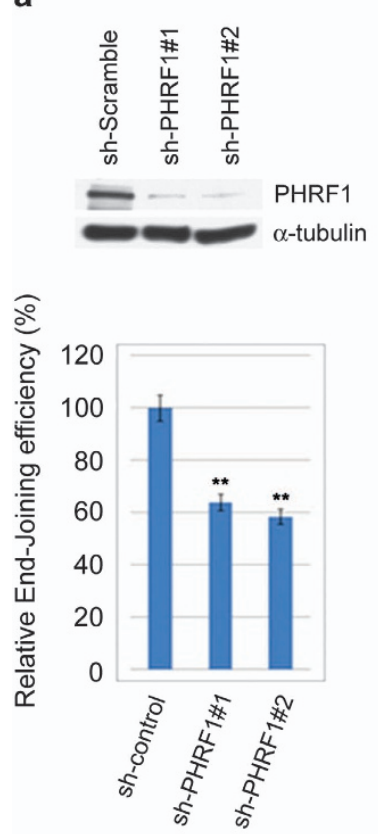

C

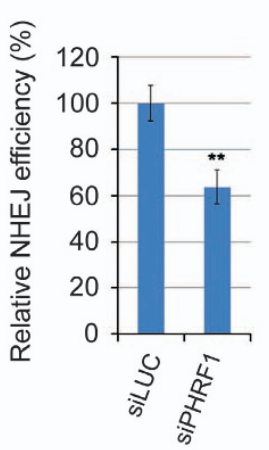

b
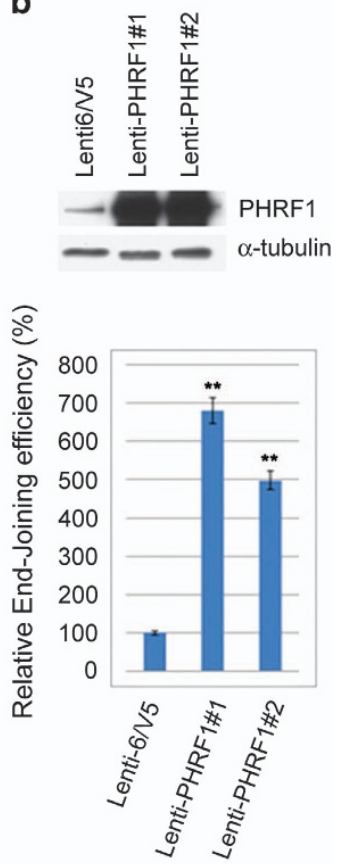

d

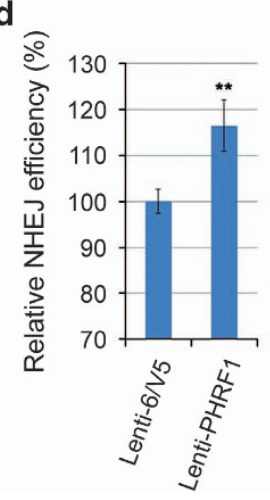

Figure 4 PHRF1 modulates NHEJ. Hindll-linearized pGL3 plasmid containing the luciferase gene was transfected into PHRF1-depleted HEK293T cells (a) and PHRF1-overexpressing U2OS cells (b), respectively. In vivo end-joining efficiency was measured by luciferase activity and normalized with $\beta$-gal expression. The data shown were mean \pm S.D. of three independent experiments. NHEJ reporter H1299dA3-1 no. 2 cells were transfected with PHRF1 siRNAs (c) or HA-PHRF1 (d) for $24 \mathrm{~h}$ and then transfected with I-Scel expression plasmid for another $48 \mathrm{~h}$. The proportion of enhanced green fluorescent protein (EGFP)-positive cells was determined by flow cytometry at $48 \mathrm{~h}$ after I-Scel transfection. Each experiment represents the mean \pm S.D. of three independent experiments. ${ }^{\star} P<0.05$ and ${ }^{* *} P<0.01$ compared with the control. HA, hemagglutinin; IP, immunoprecipitation
I-Scel transfection (Figure 5e), supporting the notion that PHRF1 and NBS1 were recruited to the sites of DSBs in vivo. Finally, SDTE mutant PHRF1 $1^{\text {S915A/S917A }}$ and PHD mutant PHRF1 $186 \mathrm{~A} / \mathrm{C} 189 \mathrm{~A}$ did not profoundly promote the NHEJ efficiency (Figure 5f), indicating that both PHD domain and SDTE motif in PHRF1 are required to promote NHEJ repair.

PHD domain and SDTE motif are essential for PHRF1 to DNA damage site. To address whether PHRF1 was recruited to DSB sites with the assistance of NBS1, NBS1 was silenced in NHEJ reporter $\mathrm{H} 1299$ cells and DSBs were generated by I-Scel digestion. ChIP results showed that HAPHRF1 was recruited to DNA damage sites only in the presence of NBS1 (Figure 6a). mCherry-NBS and GFPPHRF1 almost simultaneously appeared at DNA lesions after laser microirradiation, but mCherry-NBS1 had a longer retention time at damaged sites (Figure 6b). To determine which region in PHRF1 is responsible for its recruitment to DNA damage sites, we monitored the recruitment of GFPPHRF1 mutants to laser-induced DNA lesions. Interestingly, GFP-PHRF1 in the presence of ATM and PARP1 inhibitors and a dysfunctional E3 ligase mutant of PHRF1, GFP$\mathrm{PHRF}^{\mathrm{C} 108 \mathrm{~A}}$, were able to appear at DNA damage sites. By contrast, GFP-PHRF1 ${ }^{\text {C186A/C189A }}$ and GFP-PHRF1 ${ }^{\text {S915A/T917A }}$ did not localize to laser-irradiated regions. Additionally, the addition of TBB, a CK2 inhibitor that potentially reduces the PHRF1 ${ }^{\text {S915/T917 }}$ phosphorylation, impaired the recruitment of PHRF1 to DNA damage sites, indicating that PHD domain and phosphorylatable SDTE motif are essential for localization of PHRF1 to DNA damage sites (Figure 6b).

PHRF1 facilitates polyubiquitination of PARP1. PHRF1 contains a RING domain and this RING domain is responsible for its E3 ligase activity (Supplementary Figure S8). However, which proteins involved in NHEJ might be degraded by PHRF1 is unclear. Interestingly, the protein levels of Ku70, Ku80, NBS1, and Mre11 were mainly unchanged regardless of the expression levels of PHRF1. However, the amount of PARP1 is highly dependent on the presence of PHRF1, which was reduced in PHRF1-overexpressing HEK293T cells and increased in PHRF1-depleted HeLa cells (Figure 7a). The amount of PARP1 was restored in PHRF1 $108 \mathrm{~A}$. transfected cells, and ubiquitin ${ }^{\mathrm{K} 48 \mathrm{R}}$ was not able to incorporate into PARP1 in the presence of PHRF1, suggesting that PARP1 is ubiquitinated for proteosomal degradation by PHRF1. Indeed, the ubiquitinated PARP1 was accumulated in the presence of a proteasome inhibitor MG132 (Figure 7b), indicating that the RING domain of PHRF1 is responsible for the ubiquitination of PARP1 in vivo. To investigate whether PARP1 affected NHEJ, the protein level of PARP1 was manipulated in H1299 NHEJ reporter cells. As anticipated, NHEJ capacity was significantly compromised, while the amount of PARP1 was increased and vice versa (Figure $7 \mathrm{c}$ ). To measure alt-NHEJ efficiency, we depleted Ku70 in altNHEJ reporter EJ2-GFP U2OS cells ${ }^{29}$ and then cotransfected I-Scel and HA-PHRF1 into EJ2-GFP cells. AltNHEJ efficiency was reduced in the presence of PHRF1 compared with control HA vector (Figure 7d), indicating that PHRF1 may modulate alt-NHEJ by ubiquitinating PARP1. 


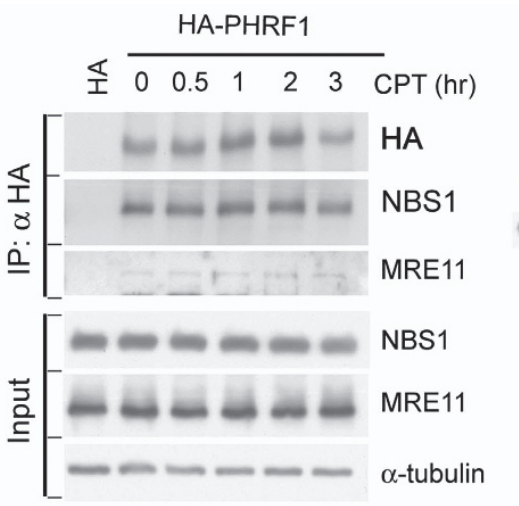

$\sum_{\substack{\infty \\ 0}}^{\bar{n}}$

d

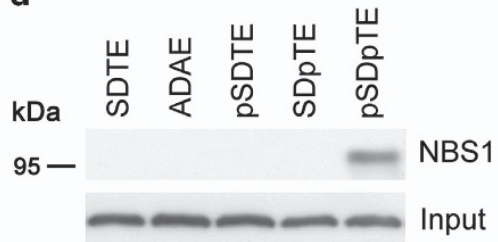

b

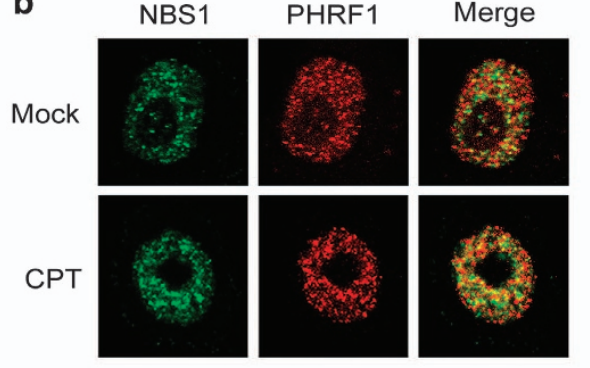

e

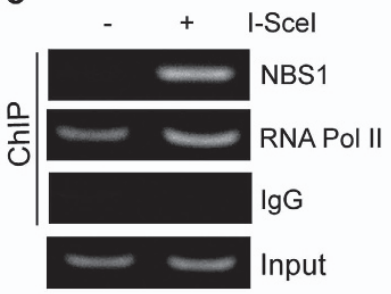

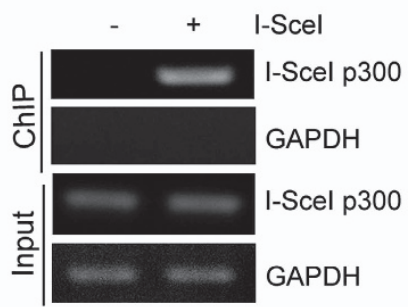

f

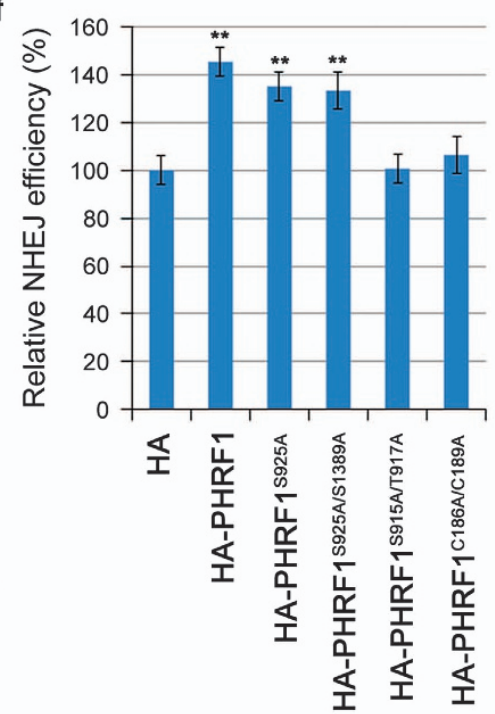

Figure 5 PHRF1 interacts with NBS1 via SDTE motif. (a) HeLa cells were transfected with empty FLAG vector and FLAG-NBS1 construct. Cell extracts were immunoprecipitated (IP) with anti-FLAG agarose and immunoblotted (IB) with anti-PHRF1 antibody. Reciprocal transfection with empty hemagglutinin (HA) vector and HA-PHRF1 was conducted. Input, the same amount of cell extracts for immunoprecipitation. (b) HeLa cells were exposed to CPT (10 $\mu \mathrm{M}$ ) for $2 \mathrm{~h}$ and then simultaneously labeled with a mixture of anti-PHRF1 mAb (red) and anti-NBS1 polyclonal antibody (green). (c) Cell extracts harvested from empty HA vector-, HA-PHRF1-, HA-PHRF1 ${ }^{\text {S925A }}$, HA-PHRF1 ${ }^{\text {S925AS1389A }}$ and HA-PHRF1 ${ }^{\text {S915ATT917A }}$-transfected HEK293T cells were IP with anti-HA agarose and IB with indicated antibodies. (d) Synthetic biotin-labeled $S^{915} \mathrm{DT}^{917} \mathrm{E}, \mathrm{A}^{915} \mathrm{DA} \mathrm{A}^{917} \mathrm{E}$, $\mathrm{pS}^{915} \mathrm{DTE}, \mathrm{SDpT}^{917} \mathrm{E}$, and $\mathrm{pS}^{915} \mathrm{DpT}^{917} \mathrm{E}$ peptides (a.a. residues $908-924$ of PHRF1) were incubated with HeLa cell extracts. IB analysis was carried out using anti-PHRF1 antibody. (e) ChIP was performed by anti-NBS1 antibody to detect the region of $0.3 \mathrm{~kb}$ downstream of I-Scel site. Control IgG for ChIP and glyceraldehyde 3-phosphate dehydrogenase (GAPDH) for PCR were as controls. (f) Empty HA vector, HA-PHRF1, HA-PHRF1 ${ }^{\text {S925A }}$, HA-PHRF1 ${ }^{\text {S925A/S1389A }}$, PHRF1 ${ }^{\text {S915AT917A }}$, and HA-PHRF1 ${ }^{\text {C186AC189A }}$ constructs were transfected into NHEJ reporter $\mathrm{H} 1299$ cells. The proportion of enhanced green fluorescent protein (EGFP)-positive cells was determined by flow cytometry at $48 \mathrm{~h}$ after I-Scel transfection. Each experiment represented the mean \pm S.D. of three independent experiments. ${ }^{\star} P<0.05$ and ${ }^{* \star} P<0.01$ compared with the control

\section{Discussion}

Our results demonstrate the involvement of PHRF1 in NHEJ repair. First, the PHD domain of PHRF1 is responsible for its binding with H3K36me2 and H3K36me3. Second, the interaction of PHRF1 with NBS1 via SDTE motif is essential for the NHEJ activity. Third, PARP1 is identified as one of the ubiquitination targets of PHRF1. Finally, on the basis of our findings, we conclude that PHRF1 may move to DSBs with the 
assistance of H3K36me2/me3 and NBS1 and then ubiquitinate PARP1 to trigger subsequent NHEJ (Figure 7e). However, the colocalization of PHRF1 with $\mathrm{H} 3 \mathrm{~K} 36 \mathrm{me} / 3$ and
NBS1 is not affected in ATM-deficient cells (Supplementary

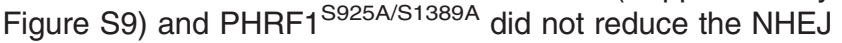
efficiency in NHEJ reporter H1299 cells (Figure 5f), suggesting

a
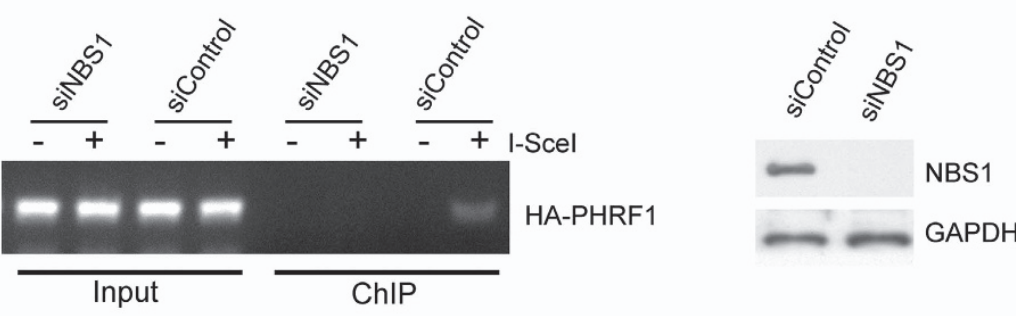

b

\section{GFP-PHRF 1}

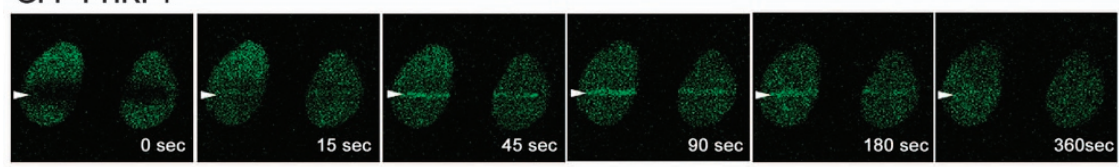

GFP-PHRF1 18186AC189A

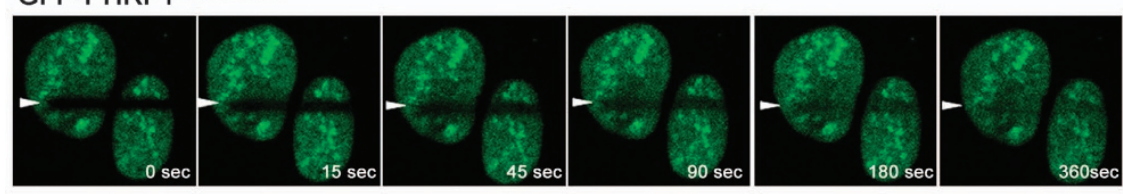

GFP-PHRF1 $1^{\text {S915AT917A }}$

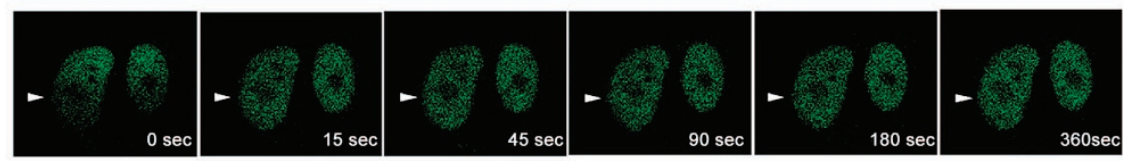

GFP-PHRF1 $108 \mathrm{~A}$

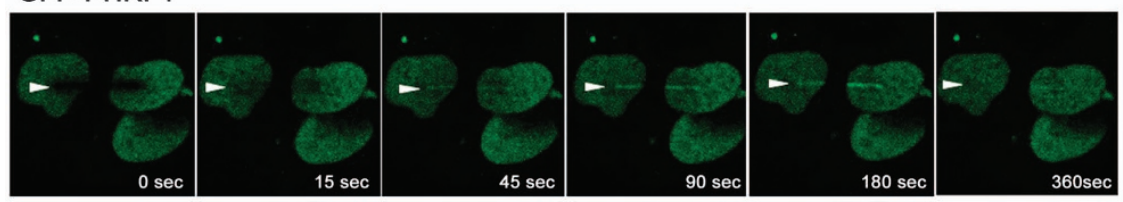

GFP-PHRF1+TBB (CK2 inhibitor)

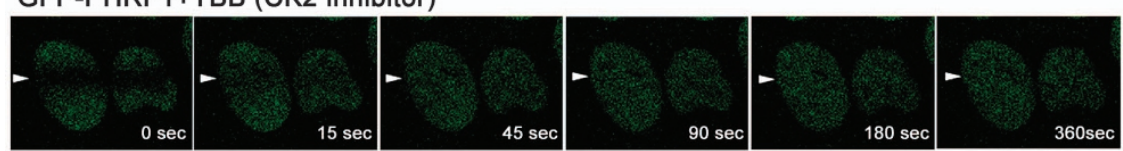

GFP-PHRF1+PJ-34 (PARP1 inhibitor)

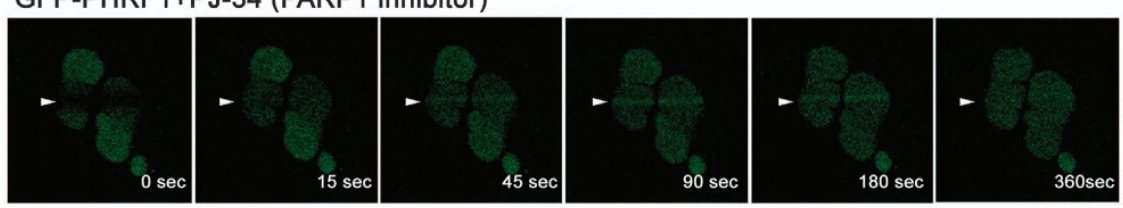

GFP-PHRF1+ATM inhibitor

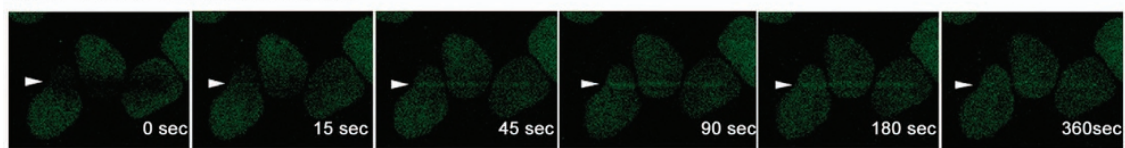

mCherry-NBS1
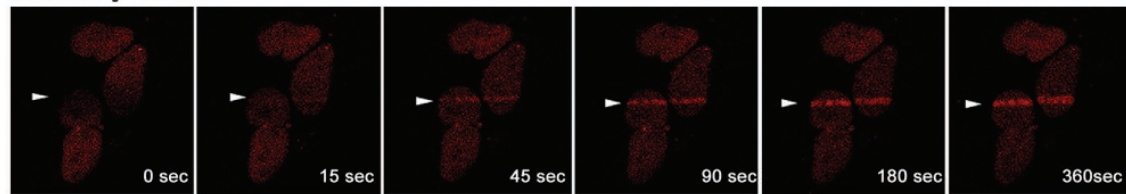
that ATM is not required for the interaction of PHRF1 with H3K36me2/3 and NBS1.

Dimethylation of H4K20me2 by histone methyltransferase MMSET is critical for the recruitment of 53BP1 to facilitate NHEJ. However, several studies fortify alternative pathways for 53BP1 recruitment to DNA damage foci. H4K16 acetylation antagonizes 53BP1 binding to H4K20me2, while H4K16 deacetylation facilitates 53BP1 foci formation and NHEJ. ${ }^{30}$ Additionally, TIP60-dependent $\mathrm{H} 4$ acetylation can diminish 53BP1 binding to H4K20me2 through the disruption of a bridge between H4K16 and Glu1551 in 53BP1. ${ }^{31}$ Although H3K36me2 near the DNA damage sites enhances the association of Ku70 and NBS1 to promote NHEJ repair, ${ }^{20}$ it is unclear whether $\mathrm{H} 3 \mathrm{~K} 36$ methylation has a direct impact for H4K20me2 to recruit 53BP1. As we did not find PHRF1 colocalization with 53BP1 and there was no significant change for 53BP1 foci formation in PHRF1-depleted and -overexpressing cells (Supplementary Figure S3), PHRF1 may not be an alternative option to link $\mathrm{H} 3 \mathrm{~K} 36 \mathrm{me} / 3$ for 53BP1 recruitment and modulate NHEJ repair.

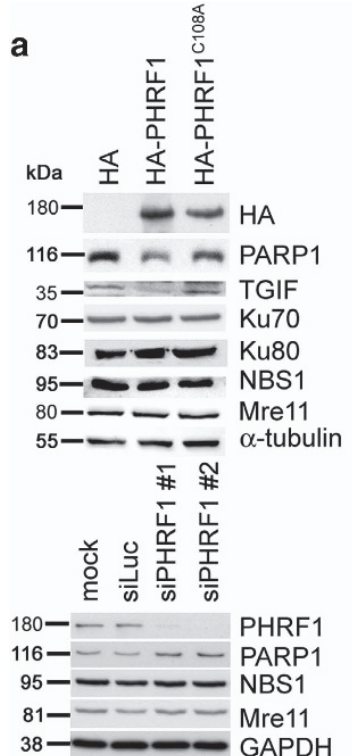

b
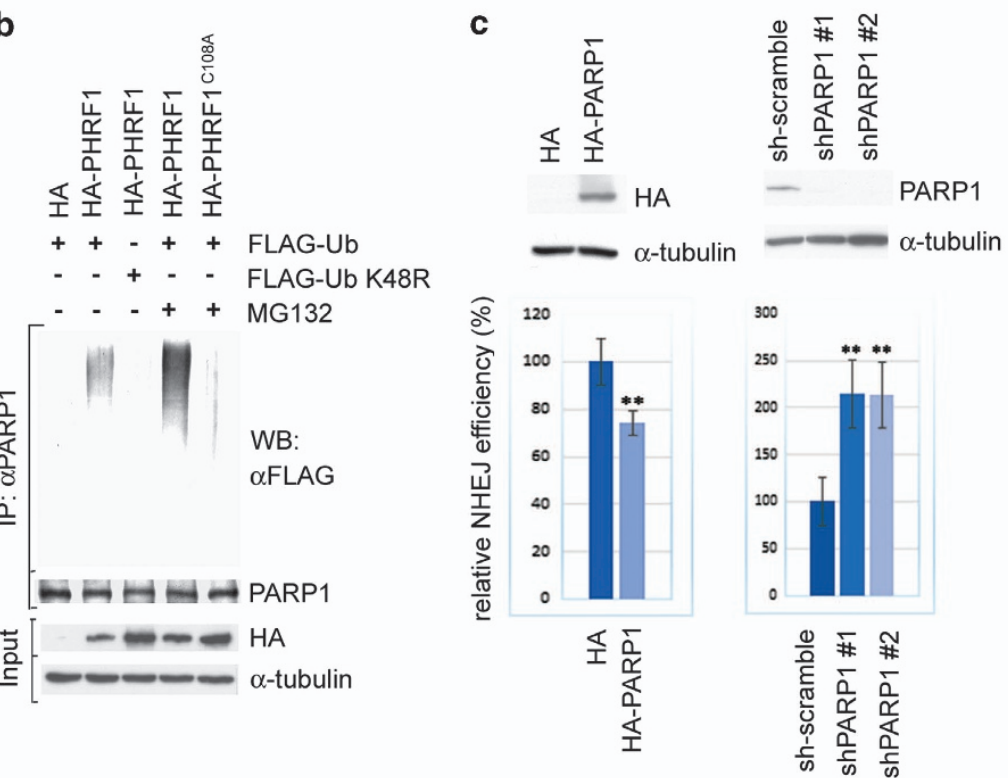

e
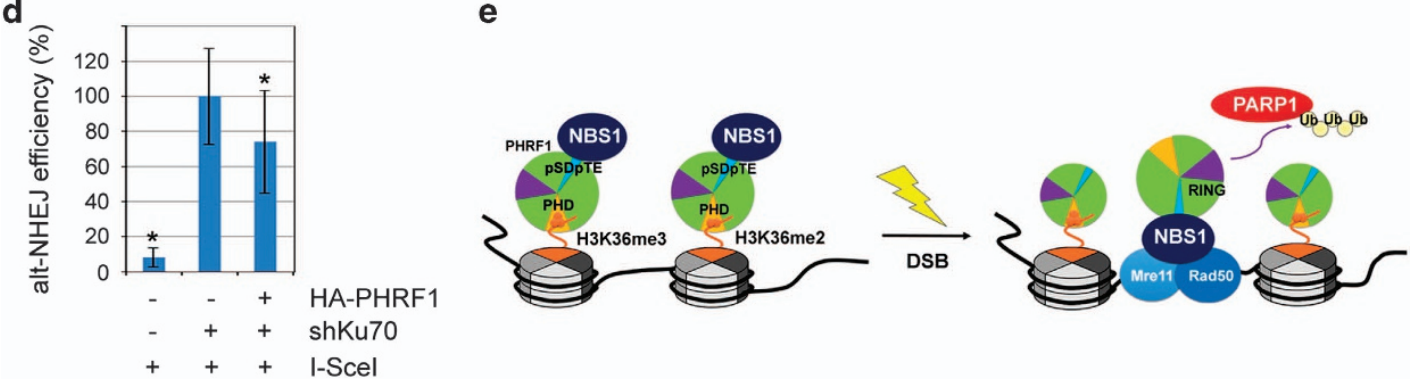

Figure 7 Poly(ADP-ribose) polymerase 1 (PARP1) is polyubiquitinated by PHRF1. (a) Total cell extracts harvested from HA-PHRF1/HA-PHRF1 $108 \mathrm{~A}$-transfected HEK293T cells and siRNA-transfected HeLa cells were immunoblotted with indicated antibodies. (b) HA-PHRF1 and HA-PHRF1 ${ }^{\mathrm{C108A}}$ were co-transfected with FLAG-Ub or FLAG-Ub ${ }^{K 48 R}$ into HEK293T cells with the addition of MG132. Total cell extracts were immunoprecipitated (IP) with anti-PARP1 antibody and immunoblotted with anti-FLAG antibody to detect ubiquitinated PARP1. (c) NHEJ reporter H1299 cells were either transfected with HA-PARP1 or PARP1 short hairpin RNA (shRNA) for $24 \mathrm{~h}$ and then transfected with I-Scel expression plasmid for another $24 \mathrm{~h}$. The proportion of enhanced green fluorescent protein (EGFP)-positive cells was determined by flow cytometry at $48 \mathrm{~h}$ after I-Scel transfection. Each experiment represented the mean \pm S.D. of three independent experiments. ${ }^{*} P<0.05$ and ${ }^{\star \star} P<0.01$ compared with the control. (d) Ku70 were depleted by shRNA in alt-NHEJ reporter EJ2-GFP cells for $48 \mathrm{~h}$ and then co-transfected with I-Scel and HA-PHRF1 or empty HA vector for another $48 \mathrm{~h}$. The proportion of GFP-positive cells was determined by flow cytometry. Each experiment represented the mean \pm S.D. of three independent experiments. ${ }^{*} P<0.05$ compared with the control. (e) A schematic model of PHRF1 in NHEJ repair. PHRF1 constitutively associates H3K36me2/3 via PHD domain and NBS1 by pSDpTE motif. When DSBs occur, PHRF1 may move to DSBs with the assistance of NBS1 to ubiquitinate PARP1 for NHEJ repair. GAPDH, glyceraldehyde 3-phosphate dehydrogenase; HA, hemagglutinin; Ub, ubiquitin; WB, western blot

Figure 6 PHD domain and SDTE motif are required for PHRF1 to DNA damage sites. (a) NBS1 was depleted in NHEJ reporter H1299 cells and then I-Scel accompanied with empty vector or HA-PHRF1 were co-transfected into $\mathrm{H} 1299$ cells. ChIP was conducted using anti-HA antibody at $48 \mathrm{~h}$ after I-Scel transfection. (b) Live cell imaging shows the dynamic recruitments of GFP-PHRF1 ${ }^{\text {C108A }}$, GFP-PHRF1 ${ }^{\text {C186AC189A }}$, GFP-PHRF1 ${ }^{\text {S915AT917A }}$, and GFP-PHRF1 in the presence of TBB (CK2 inhibitor), PJ-34 (PARP1 inhibitor), and ATM inhibitors to DNA damage sites generated by laser microirradiation in U2OS cells. mCherry-NBS1 was as a control. Note that PHRF1 and NBS1 almost simultaneously appeared at DNA damage sites before $180 \mathrm{~s}$ after laser irradiation. Arrows indicate the laser direction through the respective nuclei. GAPDH, glyceraldehyde 3-phosphate dehydrogenase; PARP1, poly(ADP-ribose) polymerase 1 
Methylation of H3K36 is generally associated with 'open' euchromatin and permissible for RNA pol II to activate gene transcription, but $\mathrm{H} 3 \mathrm{~K} 36$ methylation is also involved in alternative splicing, transcriptional repression, DNA repair, and recombination. ${ }^{25}$ Rat PHRF1 interacts with the C-terminal domain of RNA polymerase II revealed by yeast two-hybrid assay and immunoprecipitation analysis. ${ }^{32}$ Although PHRF1 preferentially associates with $\mathrm{H} 3 \mathrm{~K} 36 \mathrm{me} 3$ in active euchromatin and possibly participates in gene transcription, it is feasible that PHRF1 may assist DNA repair in euchromatin. Recently, $\mathrm{Li}$ et al. ${ }^{33}$ identified a novel role of H3K36me3 to facilitate DNA mismatch repair (MMR) by targeting the MMR machinery to chromatin. ${ }^{33}$ It has been described that MMR proteins MLH1, EXO1 and $\mathrm{MSH} 2$ are important for class-switch recombination (CSR) and are capable of converting DNA nicks and point mutations into dsDNA breaks for both C-NHEJ and alt-NHEJ pathways of CSR. ${ }^{34}$ Genetic screening of systemic sclerosis (SSc)- and systemic lupus erythematosus (SLE)-related chronic autoimmune diseases showed high frequencies of single-nucleotide polymorphisms (SNP) in IRF7/PHRF1 locus. ${ }^{35}$ Although the functional relationship between PHRF1 and SSc/SLE is unclear, it is plausible to speculate that PHRF1 may participate in V(D)J recombination or CSR, both need NHEJ to facilitate antibody production.

Our study also raises interesting questions regarding the interaction of PHRF1 with NBS1 and the ubiquitination of PARP1. MDC1 contains six SDTD clusters (a.a. 210-460) to interact with NBS1. ${ }^{26-28}$ By contrast, PHRF1 contains only one SDTE motif (a.a. 915-918). Substitution of this SDTE to ADAE in HA-PHRF1 greatly decreased its interaction with NBS1 and further impaired NHEJ efficiency (Figure 5). It would be interesting to determine under which condition or which factor directs NBS1 to choose among MDC1 and PHRF1. PARP1 is suggested to be involved in the activation of alt-NHEJ pathway ${ }^{7}$ and has been described to be ubiquitinated by CHFR and Iduna. ${ }^{36-38} \mathrm{CHFR}$ mediates polyubiquitination/ degradation of PARP1 and protects cells against DNA damage insults. Iduna is a poly(ADP-ribose)-dependent E3 ubiquitin ligase that promotes PARP1 ubiquitination and DNA repair. Is PHRF1 a redundant E3 ligase to promote PARP1 degradation or is it required for PARP1 ubiquitination under certain circumstances? We preferentially favor the notion that the recruitment of PHRF1 to DSBs is a critical phase to ubiquitinate PARP1 for NHEJ. If it is so, PHRF1 might be an alternative option by choosing the appropriate DSB repair to maintain genome integrity.

\footnotetext{
Materials and Methods

Cells lines. HEK293T cells were maintained in DMEM medium with $10 \%$ FBS. H1299dA3-1 human lung cancer cells harboring an integrated pIRES-TK-EGFP NHEJ reporter was a gift from Dr. T Kohno ${ }^{25}$ and EJ2-GFP U2OS cells was a gift from Dr. JM Stark ${ }^{29}$. U2OS cells containing DR-GFP HR reporter were cultured in McCoy's 5a medium supplemented with $10 \%$ FBS.

Antibodies. Mouse anti-PHRF1 monoclonal antibody was raised against $6 \mathrm{xHis}-$ tagged PHRF1 recombinant protein (a.a.1298-1649). Mouse anti-HA were purchased from Santa Cruz Biotech (Santa Cruz, CA, USA). Rabbit antimethylated histone antibodies were obtained from Abcam (Cambridge, UK). Mouse anti- $\alpha$-tubulin antibody was from Sigma-Aldrich (St. Louis, MI, USA). Rabbit antiNBS1, Ku70 and Ku80 antibodies were from GeneTex (Irvine, CA, USA). Rabbit anti-PARP1 antibody was from Cell Signaling (Irvine, CA, USA).
}

Small interfering RNA. The small interfering RNA (siRNA) siPHRF1 no. 1, 5'-GGACAAAGUGUUAGAGGUAdTdT-3' and siPHRF1 no. 2, 5'-CGGAAGAGCU CUAUGGGAAdTdT-3' were synthesized by GE Healthcare Dharmacon (Lafayette, CO, USA) to knockdown the expression of PHRF1.

Whole-cell extracts, nuclear extracts, and chromatin isolation. Whole-cell extracts were prepared with RIPA lysis buffer. To prepare cytosolic and nuclear extracts, $1 \times 10^{8}$ cells were lysed in buffer A (10 mM HEPES $(\mathrm{pH} 7.9)$, $10 \mathrm{mM} \mathrm{KCl}, 1.5 \mathrm{mM} \mathrm{MgCl}$, $0.34 \mathrm{M}$ sucrose, $10 \%$ glycerol, $1 \mathrm{mM} \mathrm{DTT,} 0.1 \%$ Triton $\mathrm{X}-100$, protease inhibitors). After incubation at $37^{\circ} \mathrm{C}$ for $5 \mathrm{~min}$, nuclei were lysed in $1 \mathrm{ml}$ of buffer $B$ ( $3 \mathrm{mM}$ EDTA, $0.2 \mathrm{mM}$ EGTA, $1 \mathrm{mM}$ DTT, protease inhibitors). Insoluble chromatin was separated via centrifugation ( $5 \mathrm{~min}, 2,500$ r.p.m.) and resuspended in $0.5 \mathrm{ml}$ Laemmli buffer and then sonicated for $5 \mathrm{~min}$ for SDS-PAGE.

Lentivirus production. The packaging vector pCMVdeltaR8.91, the VSV-G envelope glycoprotein vector pMD.G, and the full-length of PHRF1 in the pLenti6/V5 vector were co-transfected into HEK293 T cells. After $48 \mathrm{~h}$ incubation, the viral particles were collected to infect U2OS cells in the presence of polybrene $(8 \mu \mathrm{g} / \mathrm{ml})$ for viral infection. Stable clones were selected with blasticidine (Thermo Scientific, Waltham, MA, USA) for 21 days.

Laser microirradiation. Cells were presensitized with $10 \mu \mathrm{M}$ of 5-bromo-2deoxyuridine (BrdU; Sigma-Aldrich) for $24 \mathrm{~h}$ at $37^{\circ} \mathrm{C}$. Laser microirradiation was carried out with a Leica SP5X inverted confocal microscope (Leica Microsystems, Buffalo Grove, IL, USA) and a $405 \mathrm{~nm}$ laser diode with full output settings. Two scans were used to generate DNA damage restricted to the laser path with minimal cellular toxicity. Cells were either left untreated or in the presence of TBB $(75 \mu \mathrm{M}$; Sigma-Aldrich) for $6 \mathrm{~h}$, PJ-34 (10 $\mu \mathrm{M}$; Sigma-Aldrich) for $2 \mathrm{~h}$, or ATM inhibitor $(10 \mu \mathrm{M}$; Calbiochem, Billerica, MA, USA) for $2 \mathrm{~h}$.

Alkali comet assay. Single-cell gel electrophoretic comet assays were performed under alkaline conditions using Trevigen CometAssay Kit (Gaithersburg, MD, USA). Briefly, $2 \times 10^{5}$ cells were exposed to etoposide $(20 \mu \mathrm{M})$ for $30 \mathrm{~min}$ in HeLa cells and $1 \mathrm{~h}$ in U2OS cells, respectively. Cells were gently mixed with $1 \%$ low melting agarose at $37^{\circ} \mathrm{C}$ at the ratio of $1: 10(\mathrm{v} / \mathrm{v})$ and then spread onto slides. The slides were immersed in the alkali unwinding solution $(0.3 \mathrm{M} \mathrm{NaOH}, 1 \mathrm{mM}$ EDTA, $\mathrm{pH}>13$ ) for $1 \mathrm{~h}$ at $4^{\circ} \mathrm{C}$, subjected to electrophoresis at $20 \mathrm{~V}$ for $30 \mathrm{~min}$, and stained in DAPI (4',6-diamidino-2-phenylindole; $10 \mathrm{mg} / \mathrm{ml})$ for $15 \mathrm{~min}$. All images were recorded using a Leica fluorescence microscope.

Colony formation assay. Five thousand cells plated in 6-well plates were exposed to CPT $(2 \mu \mathrm{M})$ for $24 \mathrm{~h}$ or etoposide $(10 \mu \mathrm{M})$ for $1 \mathrm{~h}$ in PHRF1-depleted cells and CPT $(5 \mu \mathrm{M})$ for $24 \mathrm{~h}$ or etoposide $(20 \mu \mathrm{M})$ for $1 \mathrm{~h}$ in PHRF1overexpressing cells, respectively. After incubation for 10-12 days, the surviving colonies were stained with $0.25 \%$ crystal violet in $\mathrm{ddH}_{2} \mathrm{O}$. The stained colonies with diameter $>0.1 \mathrm{~mm}$ were calculated.

Peptide synthesis and in vitro pull-down assay. Biotin-labeled histone peptides (H3K36, APATGGVKKPHRYRP; H3K36me1, APATGGVK ${ }^{\text {me1 }}$

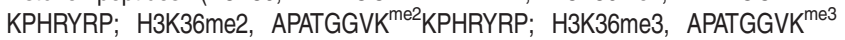
KPHRYRP; H4K20me2, GGAKRHRK ${ }^{\text {me2}}$ VLRDNIQ; H4K20me3, GGAKRHRK ${ }^{\text {me3 }}$ VLRDNIQ were synthesized by Genemed Synthesis (San Antonio, TX, USA). Biotin-labeled NBS1 peptides, SDTE (AVAAEGASDTEREEPTE; a.a. 908-924), ADAE (AVAAEGAADAEREEPTE), pSDTE (AVAAEGApSDTEREEPTE), SDPTE (AVAAEGASDpTEREEPTE), and pSDpTE (AVAAEGApSDpTEREEPTE), were synthesized at the Institute of Biological Chemistry at Academia Sinica (Taipei, Taiwan). Five hundred micrograms of HeLa whole-cell extracts were incubated with biotin-labeled peptides at $4{ }^{\circ} \mathrm{C}$ for $3 \mathrm{~h}$, and the complex was then incubated with streptavidin beads (Sigma-Aldrich) for another $3 \mathrm{~h}$. The immunoprecipitated complex were resolved by SDS-PAGE and analyzed by immunoblotting.

In vivo end-joining assay. Linearized plasmid pGL32 (Promega, Madison, WI, USA) by Hindlll was purified with phenol/chloroform extraction and ethanol precipitation, and then transfected into HEK293T cells. At $48 \mathrm{~h}$ posttransfection, luciferase activity was assayed using Bright-Glo Luciferase Assay Kit (Promega). The transfection of SV40- $\beta$-gal plasmid was used as the normalized control.

In vivo ubiquitination. HEK293T cells were co-transfected with FLAG-tagged ubiquitin and HA-tagged PHRF ${ }^{\text {WT }}$ or PHRF1 RING mutants (HA-PHRF1 ${ }^{\text {C108A }}$ and HA-PHRF1 $1^{1110 S / W 137 R}$ ) with or without the addition of MG132 proteasome inhibitor. 
Total cell extracts from transfected cells were harvested in RIPA buffer at $40 \mathrm{~h}$ after transfection for immunoblotting analysis.

\section{Conflict of Interest}

The authors declare no conflict of interest.

Acknowledgements. We are grateful to Dr. Geen-Dong Chang for critical comments on this article and indebted to Dr. Kao-Reou Wu for the gift of FLAG-NBS1. We thank Sheng-Wei Lin at Academia Sinica for technical assistances and staffs of TC5 Bio-Image Tools, College of Life Science at National Taiwan University for confocal microscopy. This work was supported by Ministry of Science and Technology (MOST 102-2311-B-002-042 and 103-2311-B-002-023) and National Taiwan University (103R7602B3) (to MSC) and in part supported by Grant EX959508NHRI from National Health Research Institute (to MDT).

1. Thompson LH. Recognition, signalling, and repair of DNA double-strand breaks produced by ionizing radiation in mammalian cells: the molecular choreography. Mut Res 2012; 751 158-246.

2. Ciccia A, Elledge SJ. The DNA damage response: making it safe to play with knives. Mol Cell 2010; 40: 179-204.

3. Escribano-Díaz C, Orthwein A, Fradet-Turcotte A, Xing M, Young JT, Tkáč J, et al. A cell cycle-dependent regulatory circuit composed of 53BP1-RIF1 and BRCA1-CtIP controls DNA repair pathway choice. Mol Cell 2013; 49: 872-883.

4. Chapman JR, Barral P, Vannier JB, Borel V, Steger M, Tomas-Loba A, et al. RIF1 is essential for 53BP1-dependent nonhomologous end joining and suppression of DNA doublestrand break resection. Mol Cell 2013; 49: 858-871.

5. Di Virgilio M, Callen E, Yamane A, Zhang W, Jankovic M, Gitlin AD, et al. Rif1 prevents resection of DNA breaks and promotes immunoglobulin class switching. Science 2013; 339: 711-715.

6. Zimmermann M, Lottersberger F, Buonomo SB, Sfeir A, de Lange T. 53BP1 regulates DSB repair using Rif1 to control 5' end resection. Science 2013; 339: 700-704.

7. Lieber MR. The mechanism of double-strand DNA break repair by the nonhomologous DNA end-joining pathway. Annu Rev Biochem 2010; 79: 181-211.

8. Stracker TH, Petrini JHT. The MRE11 complex: starting from the ends. Nat Rev Mol Cell Biol 2011; 12: 90-103.

9. Kouzarides T. Chromatin modifications and their function. Cell 2007; 128: 693-705.

10. Greer EL, Shi Y. Histone methylation: a dynamic mark in health, disease and inheritance. Nat Rev Genet 2012; 13: 343-357

11. Shi X, Hong T, Walter KL, Ewalt M, Michishita E, Hung T, et al. ING2 PHD domain links histone H3 lysine 4 methylation to active gene repression. Nature 2006; 442: 96-99.

12. Wysocka J, Swigut T, Xiao H, Milne TA, Kwon SY, Landry J, et al. A PHD finger of NURF couples histone H3 lysine 4 trimethylation with chromatin remodelling. Nature 2006; 442 $86-90$.

13. Margueron R, Justin N, Ohno K, Sharpe ML, Son J, Drury WJ III, et al. Role of the polycomb protein EED in the propagation of repressive histone marks. Nature 2009; 461 : 762-767.

14. Lachner M, O'Carroll D, Rea S, Mechtler K, Jenuwein T. Methylation of histone H3 lysine 9 creates a binding site for HP1 proteins. Nature 2001; 410: 116-120.

15. Bannister AJ, Zegerman P, Partridge JF, Miska EA, Thomas JO, Allshire RC, et al. Selective recognition of methylated lysine 9 on histone H3 by the HP1 chromo domain. Nature 2001; 410: 120-124.

16. Zhang P, Du J, Sun B, Dong X, Xu G, Zhou J, et al. Structure of human MRG15 chromo domain and its binding to Lys36-methylated histone H3. Nucleic Acids Res 2006; 34: 6621-6628.

17. Botuyan MV, Lee J, Ward IM, Kim JE, Thompson JR, Chen J, et al. Structural basis for the methylation state-specific recognition of histone $\mathrm{H} 4-\mathrm{K} 20$ by 53BP1 and Crb2 in DNA repair. Cell 2006; 127: 1361-1373.

18. Jorgensen S, Schotta G, Sorensen CS. Histone H4 lysine 20 methylation: key player in epigenetic regulation of genomic integrity. Nucleic Acids Res 2013; 41: 2797-2806.

19. Pei H, Wu X, Liu T, Yu K, Jelinek DF, Lou Z. MMSET regulates histone H4K20 methylation and 53BP1 accumulation at DNA damage sites. Nature 2011; 470: 124-128.
20. Wakeman TP, Wang Q, Feng J, Wang XF. Bat3 facilitates H3K79 dimethylation by DOT1L and promotes DNA damage-induced 53BP1 foci at G1/G2 cell-cycle phases. EMBO J 2012; 31: 2169-2181

21. Fnu S, Williamson EA, De Haro LP, Brenneman M, Wray J, Shaheen M, et al. Methylation of histone H3 lysine 36 enhances DNA repair by nonhomologous end-joining. Proc Natl Acad Sci USA 2011; 108: 540-555.

22. Matsuoka S, Ballif BA, Smogorzewska A, McDonald ER III, Hurov KE, Luo J, et al. ATM and ATR substrate analysis reveals extensive protein networks responsive to DNA damage. Science 2007; 316: 1160-1166.

23. Ettahar A, Ferrigno O, Zhang MZ, Ohnishi M, Ferrand N, Prunier C, et al. Identification of PHRF1 as a tumor suppressor that promotes the TGF-beta cytostatic program through selective release of TGIF-driven PML inactivation. Cell Rep 2013; 4: 530-541.

24. Wagner EJ, Carpenter PB. Understanding the language of Lys 36 methylation at histone $\mathrm{H} 3$. Nat Rev Mol Cell Biol 2012; 13: 115-126.

25. Ogiwara H, Ui A, Otsuka A, Satoh H, Yokomi I, Nakajima S, et al. Histone acetylation by CBP and p300 at double-strand break sites facilitates SWI/SNF chromatin remodeling and the recruitment of non-homologous end joining factors. Oncogene 2011; 30: 2135-2146.

26. Wu L, Luo K, Lou Z, Chen J. MDC1 regulates intra-S-phase checkpoint by targeting NBS1 to DNA double-strand breaks. Proc Natl Acad Sci USA 2008; 105: 11200-11205.

27. Chapman JR, Jackson SP. Phospho-dependent interactions between NBS1 and MDC1 mediate chromatin retention of the MRN complex at sites of DNA damage. EMBO Rep 2008; 9: 795-801.

28. Melander F, Bekker-Jensen S, Falck J, Bartek J, Mailand N, Lukas J. Phosphorylation of SDT repeats in the MDC1 $N$ terminus triggers retention of NBS1 at the DNA damagemodified chromatin. J Cell Biol 2008; 181: 213-226.

29. Gunn A, Bennardo N, Cheng A, Stark JM. Correct end use during end joining of multiple chromosomal double strand breaks is influenced by repair protein RAD50, DNA-dependent protein kinase DNA-PKcs, and transcription context. J Biol Chem 2011; 286: 42470-42482.

30. Hsiao KY, Mizzen CA. Histone H4 deacetylation facilitates 53BP1 DNA damage signalling and double-strand break repair. J Mol Cell Biol 2013; 5: 157-165.

31. Tang J, Cho NW, Cui G, Manion EM, Shanbhag NM, Botuyan MV, et al. Acetylation limits 53BP1 association with damaged chromatin to promote homologous recombination. Nat Struct Mol Biol 2013; 20: 317-325.

32. Yuryev A, Patturajan M, Litingtung Y, Joshi RV, Gentile C, Gebara M, et al. The C-terminal domain of the largest subunit of RNA polymerase II interacts with a novel set of serine/ arginine-rich proteins. Proc Natl Acad Sci USA 1996; 93: 6975-6980.

33. Li F, Mao G, Tong D, Huang J, Gu L, Yang W, et al. The histone mark H3K36me3 regulates human DNA mismatch repair through its interaction with MutS alpha. Cell 2013; 153: 590-600.

34. Eccleston J, Yan C, Yuan K, Alt FW, Selsing E. Mismatch repair proteins MSH2, MLH1, and EXO1 are important for class-switch recombination events occurring in B cells that lack nonhomologous end joining. J Immunol 2011; 186: 2336-2343.

35. Carmona FD, Gutala R, Simeón CP, Carreira P, Ortego-Centeno N, Vicente-Rabaneda E, et al. Novel identification of the IRF7 region as an anticentromere autoantibody propensity locus in systemic sclerosis. Ann Rheum Dis 2012; 71: 114-719.

36. Kashima L, Idogawa M, Mita H, Shitashige M, Yamada T, Ogi K, et al. CHFR protein regulates mitotic checkpoint by targeting PARP-1 protein for ubiquitination and degradation. $J$ Biol Chem 2012; 287: 12975-12984.

37. Liu C, Wu J, Paudyal SC, You Z, Yu X. CHFR is important for the first wave of ubiquitination at DNA damage sites. Nucleic Acids Res 2013; 41: 1698-1710.

38. Kang HC, Lee YI, Shin JH, Andrabi SA, Chi Z, Gagné JP, et al. Iduna is a poly(ADP-ribose) (PAR)-dependent E3 ubiquitin ligase that regulates DNA damage. Proc Natl Acad Sci USA 2011; 108: 14103-14108.

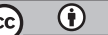

Cell Death and Disease is an open-access journal published by Nature Publishing Group. This work is licensed under a Creative Commons Attribution 4.0 International License. The images or other third party material in this article are included in the article's Creative Commons license, unless indicated otherwise in the credit line; if the material is not included under the Creative Commons license, users will need to obtain permission from the license holder to reproduce the material. To view a copy of this license, visit http://creativecommons.org/licenses/by/4.0/ 